\title{
PROPERTIES OF A DIFFERENTIAL SEQUENCE BASED UPON THE KUMMER-SCHWARZ EQUATION
}

\author{
Adhir Maharaj ${ }^{a, *}$, Kostis Andriopoulos $^{b}$, Peter Leach $^{a, b}$ \\ ${ }^{a}$ Durban University of Technology, Steve Biko Campus, Department of Mathematics, Durban, 4000, Republic of \\ South Africa \\ ${ }^{b}$ University of KwaZulu-Natal, School of Mathematical Sciences, Private Bag X54001, Durban,4000, Republic of \\ South Africa \\ * corresponding author: adhirm@dut.ac.za
}

\begin{abstract}
In this paper, we determine a recursion operator for the Kummer-Schwarz equation, which leads to a sequence with unacceptable singularity properties. A different sequence is devised based upon the relationship between the Kummer-Schwarz equation and the first-order Riccati equation for which a particular generator has been found to give interesting and excellent properties. We examine the elements of this sequence in terms of the usual properties to be investigated - symmetries, singularity properties, integrability, alternate sequence - and provide an explanation of the curious relationship between the results of the singularity analysis and a consideration of the solution of each element obtained by quadratures.
\end{abstract}

KEYWORDS: Lie symmetries, singularity analysis, differential sequence.

\section{INTRODUCTION}

In a prescient paper of 1977 Olver [1, the idea of a recursion operator for an evolution of partial differential equations, but with definite indication of extension to wider classes of partial differential equations was introduced ${ }^{1}$ Given an evolution differential equation,

$$
u_{t}=F\left(t, x, u, u_{x}, \ldots, u_{n x}\right),
$$

$R[u]$ is a recursion operator for (1) if it satisfies the equation [1] [p 1213, (8)]

$$
\left[L[F]-D_{t}, R[u]\right]_{L B} v \in\left(u_{t}-F\right),
$$

where $L[F]$ is the linearised operator

$$
L[F]=\frac{\partial F}{\partial u_{n x}} D_{x}^{n}+\ldots+\frac{\partial F}{\partial u_{x}} D_{x}+\frac{\partial F}{\partial u},
$$

$D_{t}$ and $D_{x}$ denote the total differentiation with respect to $t$ and $x$, respectively, and $v$ is a solution of the linearised equation, ie $L v=0$.

Over the last thirty years, recursion operators have found a wide application in the study of nonlinear evolution partial differential equations with particular reference to their integrability in terms of the possession of an infinite number of conservation laws.

More recently, Euler et al 4 and Petersson et al [5] examined the linearisability of nonlinear hierarchies of evolution equations in $(1+1)$ dimensions with a particular reference to the generalised $x$-hodograph transformation. As a natural development from this work, Euler et al [6] initiated a parallel study of recursion

\footnotetext{
${ }^{1}$ In this respect Olver made use of a number of results due to various authors 2 3. The beauty of the subsequent work is in his synthesis.
}

operators applied to ordinary differential equations. This was subsequently amplified by Andriopoulos et al [7] in their detailed study of the Riccati Differential Sequence $^{2}$. A further development was the introduction of the concept of an alternate sequence [8].

In this paper, we construct a first-order recursion operator for a sequence of nonlinear ordinary differential equations based upon the Schwarzian differential invariant in its expression as the Kummer-Schwarz equation and investigate the properties of the higherorder differential equations so generated. As our primary interest is in differential equations integrable in the sense of Poincaré (iewith solutions which are analytically away from isolated polelike singularities) we are disappointed with the results. However, we are able to develop a sequence based upon a different generator and find that the elements of that sequence have a combination of very interesting properties, which help to illustrate a facet of this type of analysis not displayed in the literature. In addition to the examination of the singularity properties of this sequence, we also demonstrate its complete symmetry group and provide a differential generator for an alternate representation of the elements of the sequence.

\section{THE FIRST-ORDER RECURSION OPERATOR FOR THE KUMMER-SCHWARZ EQUATION}

We write the Kummer-Schwarz equation in the form

$$
\frac{u^{\prime \prime \prime}}{2 u^{\prime}}-\frac{3 u^{\prime \prime 2}}{4 u^{\prime 2}}=0
$$

\footnotetext{
${ }^{2}$ The change in terminology is justified in that paper.
} 
where the prime denotes the differentiation with respect to the independent variable, $x$, to emphasise its connection with the Schwarzian derivative, $\left(u^{\prime-1 / 2}\right)^{\prime \prime}$.

We construct a first-order recursion operator for (4), $R=D+A$, where we write $D$ as the operator of the total differentiation with respect to the single independent variable, $x$, and $A$ is a function to be determined. The linearised operator for (4) is

$$
L[u]=\frac{1}{2 u^{\prime}} D^{3}-\frac{3 u^{\prime \prime}}{2 u^{\prime 2}} D^{2}-\left(\frac{u^{\prime \prime \prime}}{2 u^{\prime 2}}-\frac{3 u^{\prime \prime 2}}{2 u^{\prime 3}}\right) D .
$$

As we are dealing with an ordinary differential equation, (2) is somewhat simpler. We calculate the Lie Bracket of $L$ and $R$ and require its action upon $v$ to give zero when the (linearised) equation for $v$ is satisfied. This gives a large equation with terms in $v^{\prime \prime}, v^{\prime}$ and $v$. The coefficient of each of these terms is required separately to be zero. That of $v^{\prime \prime}$ gives

$$
\frac{6 A^{\prime}}{u^{\prime}}-\frac{6 u^{\prime \prime 2}}{u^{\prime 3}}+\frac{6 u^{\prime \prime \prime}}{u^{\prime 2}}=0
$$

which has the solution

$$
A=-\frac{u^{\prime \prime}}{u^{\prime}}
$$

up to an arbitrary constant of integration, which we consider to be zero. When the right side (7) is substituted into the remaining terms, the result is identically zero.

We apply the recursion operator

$$
R=D-\frac{u^{\prime \prime}}{u^{\prime}}
$$

to the Kummer-Schwarz equation, (4), to initiate the construction of the elements of the sequence. The first equation we obtain is

$$
\frac{u^{(4)}}{u^{\prime}}-5 \frac{u^{\prime \prime} u^{(3)}}{u^{\prime 2}}+9 \frac{u^{\prime \prime 3}}{2 u^{\prime 3}}=0 .
$$

When we seek the leading-order behaviour of $(9)$ by setting $u=\alpha \chi^{p}$, where $\chi=x-x_{0}$ and $x_{0}$ is the location of the putative singularity, we find that $p=-1$ (bis), 1 . This is not acceptable for a scalar equation!

We recall that the Kummer-Schwarz equation is closely related to the equation

$$
v^{\prime \prime}+v^{\prime 2}=0
$$

which is the autonomous version of the potential form of Burgers equation for which the first-order recurrence relation is $D+v^{\prime}$. This recurrence relation becomes that of the Kummer-Schwarz equation obtained above, under the transformation connecting the two equations.

Equation 10 is an elementary Riccati equation in the dependent variable $v^{\prime}$. In terms of $y=v^{\prime}$ it is

$$
y^{\prime}+y^{2}=0 \text {. }
$$

The connection of this form of the Riccati equation to the Kummer-Schwarz equation is well known. It is a simple calculation to show that the recursion operator for (11) is $D+2 y$. However, it has been shown [6, 77] that the operator, $D+y$, generates a sequence of differential equations based upon the Riccati equation, which has very satisfying properties in terms of their singularity characteristics and algebras. There is a price to pay. This operator is not a recursion operator - hence the descriptor 'generator of sequence' 7] to emphasise the distinction - and so one cannot expect to obtain the properties normally associated with the recursion operator for an hierarchy of evolution equations. However, there is much to be said for the attractive properties which we do find.

In terms of the dependent variable of the KummerSchwarz equation, the generator of the sequence, $D+y$, becomes $D-u^{\prime \prime} / 2 u^{\prime}$ and it is this operator which we employ henceforth.

\section{ThE EARLY ELEMENTS OF THE NEW SEQUENCE}

We now apply the generator

$$
R=D-\frac{u^{\prime \prime}}{2 u^{\prime}}
$$

to the Kummer-Schwarz equation, (4), to initiate the construction of the elements of the sequence and then apply it to each new element in turn. The process may be continued for as long as the memory of one's symbolic manipulator can contain the results. We simply list the first few members to give an indication of the structure of these equations. We include the first element, the original Kummer-Schwarz equation, for the sake of completeness in terms of the notation used. The KS should need no explanation. The initial ' 1 ' indicates that the sequence is generated by a firstorder operator. The two subsequent digits indicate the place of the particular equation as an element of the sequence. We obtain the following sequence,

$$
\begin{aligned}
\mathrm{KS} 101:= & \frac{u^{\prime \prime \prime}}{u^{\prime}}-\frac{3 u^{\prime \prime 2}}{2 u^{\prime 2}}=0 \\
\mathrm{KS} 102:= & \frac{u^{\prime \prime \prime \prime}}{u^{\prime}}-\frac{9 u^{\prime \prime} u^{\prime \prime \prime}}{2 u^{\prime 2}}+\frac{15 u^{\prime \prime 3}}{4 u^{\prime 3}}=0 \\
\mathrm{KS103:=} & \frac{u^{\prime \prime \prime \prime \prime}}{u^{\prime}}-\frac{6 u^{\prime \prime} u^{\prime \prime \prime \prime}}{u^{\prime 2}}-\frac{9 u^{\prime \prime \prime 2}}{2 u^{\prime 2}}+\frac{45 u^{\prime \prime 2} u^{\prime \prime \prime}}{2 u^{\prime 3}} \\
& -\frac{105 u^{\prime \prime 4}}{8 u^{\prime 4}}=0 \\
& \frac{u^{\prime \prime \prime \prime \prime \prime}}{u^{\prime}}-\frac{15 u^{\prime \prime} u^{\prime \prime \prime \prime \prime}}{2 u^{\prime 2}}-\frac{15 u^{\prime \prime \prime} u^{\prime \prime \prime \prime}}{u^{\prime 2}} \\
& +\frac{75 u^{\prime \prime 2} u^{\prime \prime \prime \prime}}{2 u^{\prime 3}}+\frac{225 u^{\prime \prime} u^{\prime \prime \prime}}{4 u^{\prime 3}} \\
& -\frac{525 u^{\prime \prime 3} u^{\prime \prime \prime}}{4 u^{\prime 4}}+\frac{945 u^{\prime \prime 5}}{16 u^{\prime 5}}=0
\end{aligned}
$$




$$
\text { KS10n: }=\quad\left(D-\frac{u^{\prime \prime}}{2 u^{\prime}}\right)^{n}\left(\frac{u^{\prime \prime \prime}}{u^{\prime}}-\frac{3 u^{\prime \prime 2}}{2 u^{\prime 2}}\right)=0 .
$$

\section{Singularity ANALYSiS}

We perform the singularity analysis in the usual fashion by firstly making the substitution

$$
u=\alpha \chi^{p},
$$

where $\chi=x-x_{0}$ and $x_{0}$ is the location of the putative singularity, to determine the possible exponents of the leading-order term and the corresponding coefficients. The results for the first few elements of the sequence are given in Table 1.

There are three points to note. Firstly, the elements of the sequence are of degree zero in $u$ and so the coefficient of the leading-order term is arbitrary. Secondly, if one removes the fractions by multiplying by the highest power of $u^{\prime}$ in the denominator, the possibility of $p=0$ has multiple occurrences. For the sequence as we have written it, these possibilities are spurious. As further analysis is more convenient to perform when the denominators are removed, we ignore these values. Thirdly, there is always the possibility that $p=1$. Such a value is without the ambit of the singularity analysis. It is amusing to note that KS101 is invariant under the transformation $u \longrightarrow 1 / u$. This property does not persist for higher elements of the sequence. The second step in the singularity analysis is to determine at what powers of $\chi$ the additional constants of integration occur. The performance of this computation is greatly facilitated by the removal of the fractions involving the derivative of the dependent variable. In Table 2, we list the resonances for the various permissible values of the exponents, $p$.

\section{Complete Symmetry Group}

The element KS101 possesses six Lie point symmetries with the algebra $\operatorname{sl}(2, R) \oplus \operatorname{sl}(2, R)$. All other elements of the differential sequence possess just four Lie point symmetries, namely $\Gamma_{1}=\partial_{x}, \Gamma_{2}=x \partial_{x}, \Gamma_{3}=\partial_{u}$ and $\Gamma_{4}=u \partial_{u}$ with the algebra $2 A_{1} \oplus 2 A_{1}$. Neither algebra is sufficient to specify the corresponding equation completely. It is necessary to have a recourse to nonlocal symmetries for the complete specification. The determination of the nonlocal symmetries is facilitated by the fact that all elements of the sequence may be linearised by means of the same transformation, which linearises the Kummer-Schwarz equation. We recall that (4) can be written as $u^{\prime 1 / 2}\left(u^{\prime-1 / 2}\right)^{\prime \prime}=0$. Consequently the linearising transformation is $u^{\prime}=1 / w^{2}$. The linear equation corresponding to the $n$th element of the sequence is ${ }^{3}$

$$
w^{(n+1)}=0
$$

for which a representation of its complete symmetry group [9, 10] is

$$
\Sigma_{i}=x^{i} \partial_{w}, i=0, n, \quad \text { and } \quad \Sigma_{n+1}=w \partial_{w} .
$$

When we reverse the transformation, the symmetries in 15 become

$\bar{\Sigma}_{i}=\left\{\int x^{i} u^{\prime 3 / 2} d x\right\} \partial_{u}, i=0, n, \quad$ and $\quad \bar{\Sigma}_{n+1}=u \partial_{u}$.

To these $n+2$ symmetries, we add the symmetry behind the reduction of order to (14), namely $\partial_{u}$. It is a simple matter to demonstrate that this indeed is a representation of the complete symmetry group. The algebra is $A_{2} \oplus_{s}(n+1) A_{1}$. The $(n+1)$-dimensional abelian subalgebra is comprised of the symmetries $\bar{\Sigma}_{i}$, $i=0, n$.

\section{The Alternate SEQUenCE}

In the Introduction, we mention the work of Euler and Leach [8] in which they demonstrated that the elements of a given sequence of ordinary differential equations of increasing order could be written in terms of equations of a lower order with a nonhomogeneous term of increasing complexity as one rose through the sequence. This is also the case with the present sequence. We illustrate the procedure with the first few elements of the sequence. We recall that the principle of the construction of the alternate sequence is based upon the relationship between one member of the sequence and the next member through the generator of sequences. The initial step is to solve the equation

$$
\left(D-\frac{u^{\prime \prime}}{2 u^{\prime}}\right) q_{1}=0
$$

Thereafter, one proceeds in a sequential manner by solving

$$
\left(D-\frac{u^{\prime \prime}}{2 u^{\prime}}\right) q_{n}=q_{n-1}, n=1, \ldots,
$$

where we adopt the convention that $q_{0}=0$. The solution of $(17)$ is

$$
q_{1}=C_{0} u^{1 / 2}
$$

where $u^{\prime-1 / 2}$ is an integrating factor for $K S 101^{4}$ The representation of the second member of the sequence

\footnotetext{
${ }^{3}$ In a usual manner of dealing with differential equations one forgets about the factor $1 / w$ corresponding to the $u^{\prime 1 / 2}$. When it comes to the generation of differential sequences, there is no place for such slackness. Were one to wish to consider the differential sequence corresponding to that of the KummerSchwarz sequence, the base equation would have to be $w^{\prime \prime} / w=$ 0 . As we see below, the removal of denominators entails an adjustment of the generator.

${ }^{4}$ This property was firstly noted in the case of recursion operators. It appears to be somewhat robust!
} 


\begin{tabular}{ll}
\hline Element identifier & Possible Exponents \\
\hline KS101 & $-1,1$ \\
\hline KS102 & $-3,-1,1$ \\
\hline KS103 & $-5,-3,-1,1$ \\
\hline KS104 & $-7,-5,-3,-1,1$ \\
\hline KS105 & $-9,-7,-5,-3,-1,1$ \\
\hline KS106 & $-11,-9,-7,-5,-3,-1,1$ \\
\hline KS107 & $-13,-11,-9,-7,-5,-3,-1,1$ \\
\hline KS108 & $-15,-13,-11,-9,-7,-5,-3,-1,1$ \\
\hline$\vdots$ & \\
\hline & $-2 \mathrm{n}+1,-2 \mathrm{n}+3,-2 \mathrm{n}+5, \ldots-7,-5,-3,-1,1$ \\
\hline
\end{tabular}

TABlE 1. Kummer-Schwarz Sequence: possible exponents of the leading-order term for the first eight elements

is then

$$
\overline{K S 102}: \quad \frac{u^{\prime \prime \prime}}{u^{\prime}}-\frac{3 u^{\prime \prime 2}}{2 u^{\prime 2}}=C_{0} u^{\prime 1 / 2} .
$$

It is an easy matter to demonstrate that in general

$$
\overline{K S 10 n}: \quad \frac{u^{\prime \prime \prime}}{u^{\prime}}-\frac{3 u^{\prime \prime 2}}{2 u^{\prime 2}}=\left(\sum_{i=0}^{n-1} \frac{1}{i !} C_{n-1-i} x^{i}\right) u^{\prime 1 / 2} .
$$

\section{Conclusion}

In the article, we have reported the results of our analysis of the elements of this sequence. It remains to interpret the results of the singularity analysis in the light of our knowledge of a route to obtain the solution of each element of the sequence in terms of a quadrature. The $n$th element of the sequence has $n$ possible values for the exponent of the leading-order term, which are acceptable, ie negative integers. As was noted in Table 1, for the first eight elements of the sequence, the precise values are $-(2 i-1)$, $i=1,2, \ldots, n$. In Table 2 , the resonances for each of the negative exponents of the leading-order term are given. Several features are to be noted. Firstly, there is the possibility of repeated resonances, which indicates the introduction of a logarithmic term into the Laurent expansion for the solution. The number of exponents of the leading-order term for which this happens increases as one proceeds down the sequence. Secondly, the highest exponent leads to a Right Painlevé Series, albeit with the intrusion of the unhappy logarithm, and the lowest exponent to what would be a Left Painlevé Series were it not for a single positive resonance equal in value to the negative of the exponent. For the exponents between the highest and the lowest, one sees a selection of both negative and positive resonances (apart from the generic -1), which indicates a full Laurent expansion, again with the possibility of logarithmic terms occurring. As should be by now well known, the Right Painlevé Series is a Laurent expansion in the neighbourhood of the singularity convergent on a punctured disc and the Left Painlevé Series has the nature of an asymptotic expansion in that it exists on the exterior of a disc centred on the singularity. The full series is defined over an annulus centred on the similarity and the existence of multiple instances of these series indicates a succession of annuli, the bounding circles of which are defined by successive singularities. Thirdly, the value of the highest resonance shows some variability. For patterns of resonances, in which there is no repeated resonance or the repeated resonance is the highest resonance, the highest resonance is the negative of the exponent of the leading-order term. For the other patterns of the resonances - always starting from the more positive exponents - this value is exceeded to the extent that it is necessary to provide a full number of constants of integration.

Evidently, some explanation of these features is required! Fortunately, we are in a position to describe the solution of each element of the sequence due to the property of the linearisation noted in $\S 5$. To provide the explanation, we make use of the third element of the sequence, which has the double merits of featuring in almost every property (the repetition of logarithms is not one of them) mentioned in the previous paragraph.

The third element of the sequence, KS103,

$$
\frac{u^{\prime \prime \prime \prime \prime}}{u^{\prime}}-\frac{6 u^{\prime \prime} u^{\prime \prime \prime \prime}}{u^{\prime 2}}-\frac{9 u^{\prime \prime \prime 2}}{2 u^{\prime 2}}+\frac{45 u^{\prime \prime 2} u^{\prime \prime \prime}}{2 u^{\prime 3}}-\frac{105 u^{\prime \prime 4}}{8 u^{\prime 4}}=0,
$$

takes the linear form

$$
w^{(4)}=0
$$

under the transformation $u^{\prime} \longrightarrow w^{-2}$. The solution of 23$)$ is

$$
w(x)=P_{3}(x),
$$




\begin{tabular}{|c|c|c|}
\hline Element identifier & Exponents & Resonances \\
\hline KS101 & -1 & $-1,0,1$ \\
\hline KS102 & $\begin{array}{l}-1 \\
-3\end{array}$ & $\begin{array}{l}-1,0,1 \text { (bis) } \\
-2,-1,0,3\end{array}$ \\
\hline KS103 & $\begin{array}{l}-1 \\
-3 \\
-5\end{array}$ & $\begin{array}{l}-1,0,1 \text { (bis), } 2 \\
-2,-1,0,1,3 \\
-3,-2,-1,0,5\end{array}$ \\
\hline KS104 & $\begin{array}{l}-1 \\
-3 \\
-5 \\
-7\end{array}$ & $\begin{array}{l}-1,0,1 \text { (bis), } 2,3 \\
-2,-1,0,1,2,3 \\
-3,-2,-1,0,1,5 \\
-4,-3,2,-1,0,7\end{array}$ \\
\hline KS105 & $\begin{array}{l}-1 \\
-3 \\
-5 \\
-7 \\
-9\end{array}$ & $\begin{array}{l}-1,0,1 \text { (bis), } 2,3,4 \\
-2,-1,0,1,2,3 \text { (bis) } \\
-3,-2,-1,0,1,2,5 \\
-4,-3,-2,-1,0,1,7 \\
-5,-4,-3,-2,-1,0,9\end{array}$ \\
\hline KS106 & $\begin{array}{l}-1 \\
-3 \\
-5 \\
-7 \\
-9 \\
-11\end{array}$ & $\begin{array}{l}-1,0,1 \text { (bis), } 2,3,4,5 \\
-2,-1,0,1,2,3 \text { (bis), } 4 \\
-3,-2,-1,0,1,2,3,5 \\
-4,-3,-2,-1,0,1,2,7 \\
-5,-4,-3,-2,-1,0,1,9 \\
-6,-5,-4,-3,-2,-1,0,11\end{array}$ \\
\hline KS107 & $\begin{array}{l}-1 \\
-3 \\
-5 \\
-7 \\
-9 \\
-11 \\
-13\end{array}$ & $\begin{array}{l}-1,0,1 \text { (bis), } 2,3,4,5,6 \\
-2,-1,0,1,2,3 \text { (bis), } 4,5, \\
-3,-2,-1,0,1,2,3,4,5 \\
-4,-3,-2,-1,0,1,2,3,7 \\
-5,-4,-3,-2,-1,0,1,2,9 \\
-6,-5,-4,-3,-2,-1,0,1,11 \\
-7,-6,-5,-4,-3,-2,-1,0,13\end{array}$ \\
\hline KS108 & $\begin{array}{l}-1 \\
-3 \\
-5 \\
-7 \\
-9 \\
-11 \\
-13 \\
-15\end{array}$ & $\begin{array}{l}-1,0,1 \text { (bis), } 2,3,4,5,6,7 \\
-2,-1,0,1,2,3 \text { (bis), } 4,5,6 \\
-3,-2,-1,0,1,2,3,4,5 \text { (bis) } \\
-4,-3,-2,-1,0,1,2,3,4,7 \\
-5,-4,-3,-2,-1,0,1,2,3,9 \\
-6,-5,-4,-3,-2,-1,0,1,2,11 \\
-7,-6,-5,-4,-3,-2,-1,0,1,13 \\
-8,-7,-6,-5,-4,-3,-2,-1,0,15\end{array}$ \\
\hline
\end{tabular}

TABLE 2. Kummer-Schwarz Sequence: Resonances for the permissible values of the exponent of the leading-order term 
where $P_{3}(x)$ is a polynomial of degree three in $x$. Consequently, the solution of 22 can be written in terms of the quadrature,

$$
u(x)=\int \frac{d x}{P_{3}(x)^{2}}+M .
$$

The evaluation of the quadrature in 25 is, in principle, a simple matter due to the Fundamental Theorem of Algebra and the use of partial fractions. However, the interpretation of the results of the singularity analysis obtained above requires that the quadrature is to be approached with a certain degree of delicacy to illustrate the different possibilities. We commence with the case $p=-1$ and write the solution in terms of a polynomial about the singularity at $x_{0}$ in terms of the variable $\chi=x-x_{0}$. We have

$$
P_{3}(x)=K \chi(\chi-a)(\chi-b),
$$

where we have written the factors in the sense that $0<|a| \leq|b|$ to empathise that we are dealing with a simple pole at $x_{0}$. When we substitute (26) into (25), we obtain

$$
u(x)=\int \frac{d x}{(K \chi(\chi-a)(\chi-b))^{2}}+M .
$$

We may write the integrand of (27) as

$$
\frac{1}{K^{2} \chi^{2} a^{2} b^{2}}\left(1-\frac{\chi}{a}\right)^{-2}\left(1-\frac{\chi}{b}\right)^{-2} \text {. }
$$

After applying the binomial expansion and simplifying, we may write 28 as

$$
\begin{aligned}
& \frac{1}{K^{2} a^{2} b^{2}}\left[\frac{1}{\chi^{2}}-2\left(\frac{1}{a}+\frac{1}{b}\right) \frac{1}{\chi}+\left(\frac{3}{a^{2}}+\frac{3}{b^{2}}+\frac{4}{a b}\right)\right. \\
& \left.+2\left(\frac{2}{a^{3}}+\frac{2}{b^{3}}-\frac{3}{a b^{2}}-\frac{3}{a^{2} b}\right) \chi \cdots\right] .
\end{aligned}
$$

When we substitute 29 into 27 and perform the quadrature, we obtain

$$
\begin{aligned}
u(x)= & M-\frac{1}{K^{2} a^{2} b^{2}}\left[\frac{1}{\chi}+2\left(\frac{1}{a}+\frac{1}{b}\right) \log \chi\right. \\
& -\left(\frac{3}{a^{2}}+\frac{3}{b^{2}}-\frac{4}{a b}\right) \chi \\
& \left.-\left(\frac{2}{a^{3}}+\frac{2}{b^{3}}-\frac{3}{a b^{2}}-\frac{3}{a^{2} b}\right) \chi^{2} \ldots\right]
\end{aligned}
$$

The occurrence of the unfortunate logarithm and the requisite number of constants of integration (five in this case, $K, a, b, x_{0}$ and $\left.M\right)$ is obvious. The quadrature evaluated above is valid to the next singularity at $\chi=a$.

For the case $p=-5$, we may write the integrand of 27) as

$$
\frac{1}{k^{2} \chi^{6}}\left(1-\frac{a}{K}\right)^{-2}\left(1-\frac{b}{K}\right)^{-2} .
$$

After the application of the binomial expansion and simplification of 31 , we obtain

$$
\begin{aligned}
& \frac{1}{k^{2} \chi^{6}}\left[1-\frac{2(a+b)}{\chi}+\frac{3\left(a^{2}+b^{2}\right)+4 a b}{\chi^{2}}\right. \\
& \left.-\frac{4\left(a^{3}+b^{3}\right)+6 a^{2} b^{2}+6 a b^{2}}{\chi^{3}} \ldots\right] .
\end{aligned}
$$

When we substitute 32 into 27 and perform the quadrature, we have

$$
\begin{aligned}
u(x)= & \frac{1}{K^{2}}\left[-\frac{1}{5 \chi^{5}}+\frac{(a+b)}{4 \chi^{6}}-\frac{3\left(a^{2}+b^{2}\right)+4 a b}{7 \chi^{7}}\right. \\
& \left.+\frac{2\left(a^{3}+b^{3}\right)+3 a^{2} b+3 a b^{2}}{4 \chi^{8}} \ldots\right]+M, \quad(33
\end{aligned}
$$

where the requisite number of constants of integration is also obvious.

Finally, we consider the case $p=-3$ where the full series is defined over an annulus centred on the singularity and we write the factors in the sense that $|a|<|\chi|<|b|$. We now write integrand of 27] as

$$
\frac{1}{K^{2} b^{2} \chi^{4}}\left(1-\frac{a}{\chi}\right)^{-2}\left(1-\frac{\chi}{b}\right)^{-2} .
$$

By applying the above method to (34) we obtain

$$
\begin{aligned}
u(x)= & M+\frac{1}{b^{2} K^{2}}\left[\left(-\frac{4}{b^{3}} \ldots\right) \log \chi-\frac{1}{\chi}\left(\frac{3}{b^{2}}+\frac{8 a}{b^{3}} \ldots\right)\right. \\
& -\frac{1}{2 \chi^{2}}\left(-\frac{2}{b}-\frac{6 a}{b^{2}}-\frac{12 a^{2}}{b^{3}} \ldots\right) \\
& -\frac{1}{3 \chi^{3}}\left(1+\frac{4 a}{b}+\frac{9 a^{2}}{b^{2}}+\frac{16 a^{3}}{b^{3}} \ldots\right) \\
& -\frac{1}{4 \chi^{4}}\left(-2 a-\frac{6 a^{2}}{b}-\frac{12 a^{3}}{b^{2}} \ldots\right) \\
& \left.-\frac{1}{5 \chi^{5}}\left(3 a^{2}+\frac{8 a^{3}}{b} \ldots\right)\right] .
\end{aligned}
$$

We observe that 35 contains an unfortunate logarithmic term, which is not indicated in the resonances for $p=-3$. This is possible since we have a complete Laurent series, which must necessarily be convergent in an annulus centred on the singularity. The KummerSchwarz equation and the members of the sequence generated from it by the generator 12 can be easily integrated. What we have done here is to explore its properties from a wider perspective so that a greater appreciation of its properties can be gained. The exploration of different aspects of such sequences adds to our understanding of them.

\section{ACKNOWLEDGEMENTS}

PGLL would like to thank the National Research foundation of the Republic of South Africa, University of KwaZulu-Natal and Durban University of Technology for their continued support. AM would like to thank the Durban University of Technology for their continued support. 


\section{REFERENCES}

[1] P. J. Olver. Evolution equations possessing infinitely many symmetries. Journal of Mathematical Physics 18:1212 - 1215, 1977. DOI:10.1063/1.523393

[2] C. S. Gardner. Korteweg-de vries equation and generalisations IV. The Korteweg-de Vries equation as a Hamiltonian system. Journal of Mathematical Physics 12:1548 - 1551, 1971. DOI:10.1063/1.1665772.

[3] I. M. Gelfand, L. A. Dikii. Asymptotic behaviour of the resolvent of Sturm-Liouville equations and the algebra of the Korteweg-de Vries equations. Russian Mathematical Surveys 30:77 - 113, 1975. DOI:10.1070/RM1975v030n05ANEH001522

[4] M. Euler, N. Euler, N. Petersson. Linearizable hierarchies of evolution equations in $(1+1)$ dimensions. Studies in Applied Mathematics 111:315 - 337, 2003. DOI:10.1111/1467-9590.t01-1-00236

[5] N. Petersson, N. Euler, M. Euler. Recursion operators for a class of integrable third-order evolution equations. Journal of Mathematical Physics 112:201 - 225, 2004. DOI:10.111/j.0022-2526.2004.02511.x
[6] M. Euler, N. Euler, P. G. L. Leach. The riccati and ermakov-pinney hierarchies. Journal of Nonlinear Mathematical Physics 14:290 - 310, 2007. DOI:10.2991/jnmp.2007.14.2.11.

[7] K. Andriopoulos, P. G. L. Leach, A. Maharaj. On differential sequences. Applied Mathematics and Information Sciences - An International Journal 5(3), 2011.

[8] N. Euler, P. G. L. Leach. Aspects of proper differential sequences of ordinary differential equations. Theoretical and Mathematical Physics 159:473 - 486, 2009. DOI:10.1007/s11232-009-0038-y.

[9] K. Andriopoulos, P. G. L. Leach, G. P. Flessas. Complete symmetry groups of ordinary differential equations and their integrals: some basic considerations. Journal of Mathematical Analysis and Applications 262:256 - 273, 2001. DOI:10.1006/jmaa.2001.7570.

[10] K. Andriopoulos, P. G. L. Leach. The economy of complete symmetry groups for linear higher dimensional systems. Journal of Nonlinear Mathematical Physics 9(S-2), 2002. 